\section{Perspectivas da Teoria Ator-Rede na Adesão ao Tratamento Medicamentoso por Idosos}

\author{
Actor-Network Theory's Prospects in Accession to Medicinal Treatment \\ by Elderly
}

Luiz Henrique Chenchi ${ }^{1}$, Lídia Bonfanti Anitelli², Wilson José Alves Pedro ${ }^{3}$

\section{RESUMO}

O envelhecimento populacional importa instigações para as áreas de ciência, tecnologia, educação e saúde, de modo a garantir autonomia e longevidade à pessoa idosa. Um destes desafios consiste em efetivar a adesão ao tratamento farmacológico nesta faixa etária, visto que a utilização de vários fármacos é frequente e pode acarretar complicações ao bemestar social. Em um recorte especulativo recorreu-se à Teoria Ator-Rede (ANT) com o objetivo de obter um aporte para elucidar os agentes envolvidos nessa trama e apreender seus movimentos. Empregou-se de levantamento bibliográfico, tanto do conteúdo da disciplina Metodologia e Teorias Interdisciplinares Aplicadas ao Campo CTS do Programa de Pós-graduação em Ciência, Tecnologia e Sociedade da Universidade Federal de São Carlos do ano de 2016, da qual esta abordagem é fruto, quanto dos empregos dos descritores "Saúde do Idoso" e "Adesão ao Medicamento" na base de dados Scielo. Os resultados permitiram tratar dos aspectos norteadores que sustentam uma rede de terapia farmacológica em sujeitos da terceira idade, como os profissionais implicados, aparatos tecnológicos, ambiente social e em seguida prospectou as perspectivas teóricas para construção do cuidado. Foi constatada uma diversidade de actantes e de outras redes que se interligam, as quais são essenciais para adesão ao tratamento medicamentoso por idosos, que ponderados à concepção da ANT propiciam a garantia efetiva da assistência integral por direitos, dentre os quais à saúde.

Palavras-chave: Ciência. Tecnologia e Sociedade. Adesão à Medicação. Saúde do Idoso.

\section{ABSTRACT}

Population aging implies instigations for the areas of science, technology, education and health, in order to guarantee autonomy and longevity for the elderly. One of these challenges is to achieve adherence with pharmacological treatment in this age group, since the use of several drugs is frequent and can lead to complications to social well-being. In a speculative cut, the actor-network theory (ANT) was used in order to obtain a contribution to elucidate the agents involved in this plot and to apprehend its movements. A bibliographical survey of the content of the discipline Methodology and Interdisciplinary Theories Applied to the CTS Field of the Postgraduate Program in Science, Technology and Society of the Federal University of São Carlos of the year 2016 was used and of the uses of the descriptors "Health of the Elderly" and "Adherence to the Medication" in the Scielo database. The results allowed to deal with the guiding aspects that support a network of pharmacological therapy in elderly subjects, such as involved professionals, technological apparatus, social environment and then prospect the theoretical perspectives for the construction of care. A diversity of actants and other interconnected networks were found, which are essential for adherence to drug treatment by the elderly, who, weighted to the ANT conception, provide an effective guarantee of full rights assistance, including health care.

Keywords: Science. Technology and Society. Adhesion to Medication. Health of the Elderly.
${ }^{1}$ UFSCar.

E-mail: Ihchenchi@gmail.com

${ }^{2}$ UFSCar.

${ }^{3}$ UFSCar. 


\section{INTRODUÇÄAO}

Dados do Instituto Brasileiro de Geografia e Estatística (IBGE), 2013, estimam que mais de 22 milhões de pessoas no Brasil estão com 60 anos ou mais, representando, em porcentagem, 10,98\% do total da população brasileira. Isso se deve ao fato do desenfreado crescimento da faixa etária idosa, principalmente após a década de 70 , quando a expectativa de vida começou a aumentar e a taxa de natalidade cair. O grupo de idosos aumentará acentuadamente, passando de 13,8\% em 2020 para 33,7\% em 2060, significando um aumento de $20 \%$ desta camada da população (BRASIL, 2013).

Estudos de Cintra et al. (2010), Sousa et al. (2011) e Tavares et al. (2013) apontam que os idosos são usuários de elevado número de medicamentos e que vários fatores interferem na adesão ao tratamento farmacológico, dentre os quais: aspectos relacionados a patologia (como gravidade dos sintomas, nível de deficiência, progressão e severidade, disponibilidade do tratamento), consumo em grandes quantidades e uso por longos períodos (cronicidade), dificuldade de deglutição, efeitos indesejáveis, sintomas desaparecerem, desconhecimento sobre os medicamentos, valor financeiro do tratamento, falta de motivação, ingestão de bebidas alcoólicas, analfabetismo, distúrbios de memória, automedicação, interação com profissional de saúde e a simultaneidade com outras condições de saúde que requeiram mais fármacos.

Esses aspectos podem impactar em baixa, inadequada ou nenhuma adesão à farmacologia prescrita, podendo levar a esta parcela da população aumento da demanda nos serviços de saúde e contribuir para internação hospitalar. Cintra et al. (2010, p. 3508) alerta que:

Os problemas decorrentes da não adesão ao tratamento medicamentoso aumentaram nas últimas décadas e têm preocupado os profissionais de saúde, dada a participação de fatores que influenciam o uso racional dos recursos terapêuticos. Há quase duas décadas, já se chamava a atenção para a complexidade do uso inapropriado dos medicamentos entre os idosos, e destacavase a importância de os profissionais de saúde orientarem os pacientes sobre o uso correto das drogas.

No paciente geriátrico, a tomada de um único medicamento pode gerar vários efeitos sistêmicos, devido às alterações funcionais de órgãos importantes como fígado, rins, coração e do comprometimento da vascularização, o que incrementa as possibilidades de 
interações com outras substâncias, alimentos e álcool, além de exacerbar ou atenuar a resposta do organismo a alguns fármacos, o que pode acarretar prejuízos operacionais e comprometer a qualidade de vida (BRASIL, 2012; SOUSA et al., 2011).

Em Tavares et al. (2013) tem-se que aderir ao tratamento é a harmonização entre a prescrição médica ou orientações de outro profissional da saúde e o comportamento do indivíduo, que em baixo grau compromete o prognóstico e traz consequências pessoais, sociais e econômicas.

Ao pensar CTS (Ciência, Tecnologia e Sociedade) caminhos que visem minimizar os elementos que permeiam a não aderência à terapia medicamentosa pela pessoa idosa é que se faz referência à concepção dos estudos sociais da ciência e da tecnologia, a partir da década de 1970, de maneira especial com Bruno Latour e Michel Calloncom o método construtivista, onde as coisas, fatos, pensamentos são elucidados através construções coletivas. Para esses autores, tais construções se desenvolvem em redes, onde há interação entre atores humanos e não humanos e ambos são igualmente importantes na estrutura e manutenção da rede, configurando a chamada Teoria Ator-Rede (Actor-Network Theory - ANT).

De modo a contribuir nos aspectos que envolvem a adesão ao tratamento medicamentoso por idosos, este trabalho objetiva refletir sobre desdobramentos da teoria ator-rede aplicados aos fatores relacionados à adesão ou não à terapia medicamentosa por idosos e apontar as perspectivas teóricas que contribuam para efetivação desta adesão.

\section{METODOLOGIA}

Empregou-se de levantamento bibliográfico para subsidiar o entendimento da rede de atores envoltos à terapia farmacológica por idosos, além dos fatores intrínsecos e extrínsecos nesse processo.

Para tal utilizou-se do aporte teórico acerca da ANT desenvolvido na disciplina Metodologia e Teorias Interdisciplinares Aplicadas ao Campo CTS pelo Programa de Pósgraduação em Ciência, Tecnologia e Sociedade da Universidade Federal de São Carlos no ano de 2016 que abarcava sobre a construção social do conhecimento vista por Latour. 
$\mathrm{Na}$ tentativa de construir um modelo de cuidado que auxilie e efetive a aderência ao tratamento realizaram-se buscas na literatura por artigos empregando-se os descritores "Saúde do Idoso" e "Adesão ao Medicamento" na base de dados Scielo (Scientific Electronic Library Online), a qual engloba uma variedade de periódicos das mais diversas áreas do conhecimento, permitindo um enfoque interdisciplinar desejado para os achados.

Mesmo não integrando o escopo de descritores da saúde segundo o DeCS (Descritores em Ciências da Saúde) adicionou-se o termo Teoria Ator-Rede ou ANT nas buscas para vislumbrar trabalhos que a envolvessem em seu modo de pesquisar saúde.

Foi delimitado o período dos últimos 10 anos por considerar que as questões acerca do cuidado à saúde no envelhecimento se destacarem com a longevidade da população.

Não se revelaram estudos que permeassem entre todos os descritores, o que evidenciou a necessidade desta abordagem. Os textos selecionados apontaram de alguma forma para os condicionantes ligados à adesão ao tratamento farmacológico na população idosa e o emprego da ANT veio como caminho para prospectar essa compreensão.

\section{RESULTADOS}

Os dados levantados permitiram a configuração de duas conjecturas a serem discutidas: os agentes envolvidos no tratamento farmacológico do idoso e a ótica da construção do cuidado.

\section{Proposições da rede terapêutica medicamentosa do idoso}

A conquista social do aumento da expectativa de vida da população é acompanhada por grandes desafios para as políticas públicas mundiais. No Brasil houve uma estruturação em relação ao envelhecimento populacional através da Política Nacional do Idoso (1994) e Estatuto do Idoso (2003), que asseguram direitos a este grupo etário e, especificamente na área da saúde, com a Política Nacional de Saúde da Pessoa Idosa e o Pacto de Saúde, que em 2006, priorizam a saúde da população idosa (SANTOS et al., 2016).

Dentre várias ações impostas para efetividade da saúde do idoso destaca-se a realização de uma adequada terapia medicamentosa. Existem vários aspectos que permeiam a adesão ou não ao tratamento quando se trata deste grupo etário, o que estabelece a constituição uma rede de atores envolvidos na trajetória da pessoa idosa ao utilizar os serviços em uma unidade de saúde e sair com receituário indicativo para o uso de fármacos (Figura 1). 
Os fatores, neste percurso, que determinam a adesão incluem a execução de maneira equilibrada, clareza nas orientações e dispensação, vontade e capacidade próprias de cumprir as recomendações, satisfação com o serviço e equipe de saúde, custo, quantidade e disponibilidade dos medicamentos. Ainda pode-se associar o avanço da idade e o déficit cognitivo com a não adesão à terapia, que implicariam na habilidade de leitura das bulas, compreensão e seguimento das instruções, entendimento da linguagem, incorporação à rotina diária, manejos quando esquecida a ingestão de uma dose, planejamento para acesso aos fármacos (AIOLFI et al., 2015).

Estabelecida uma a rede de cuidado ao idoso, principalmente no que remete à adesão ao tratamento farmacológico, faz-se imprescindível o fortalecimento e estreitamento das relações entre as redes, sobretudo através do repasse de informações, "diretamente relacionado ao envolvimento e integração entre os profissionais de saúde que constroem o sistema público de saúde brasileiro" (p. 59), que balizem o acompanhamento do idoso e garanta uma atenção integral (SANTOS et al., 2016).

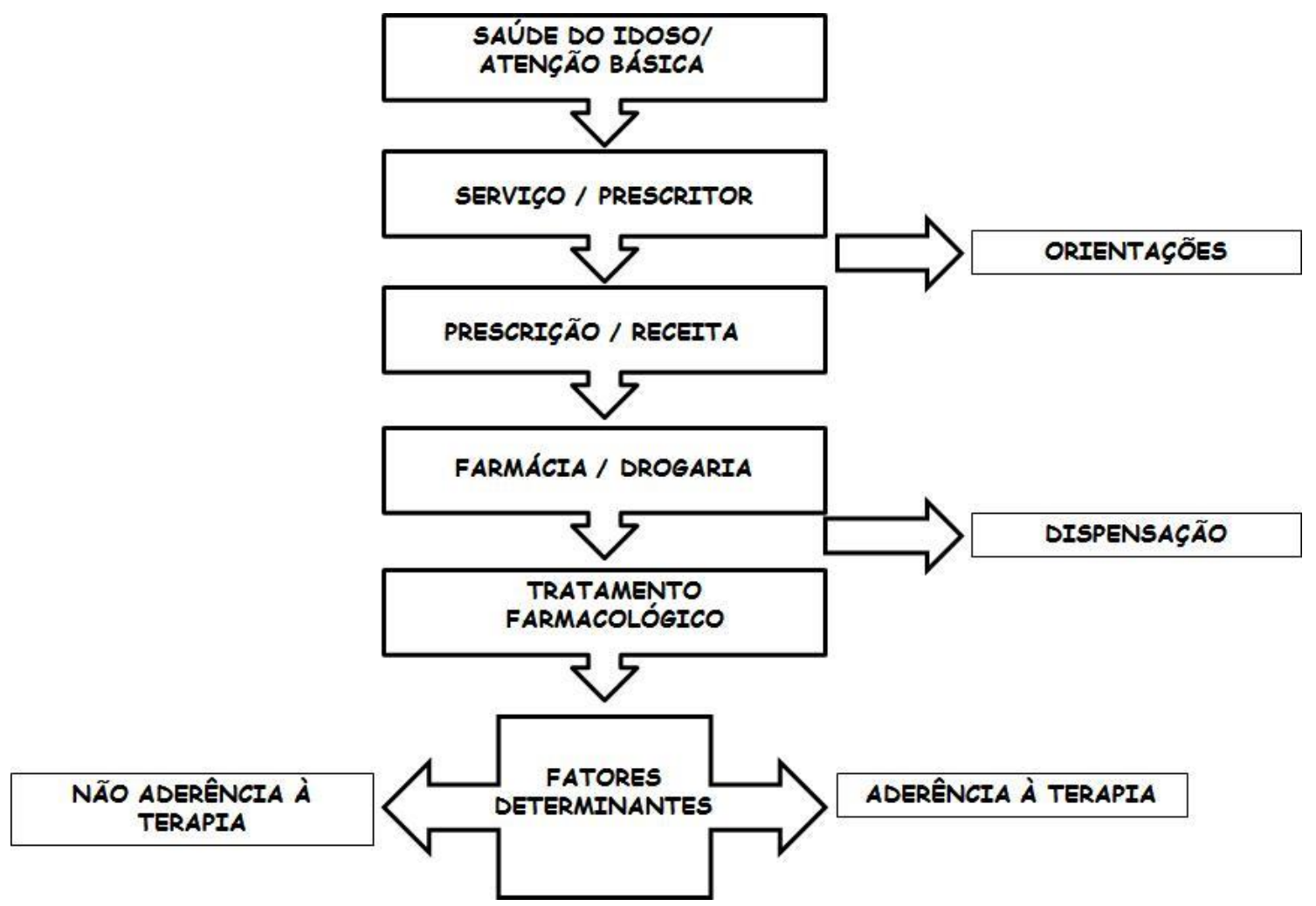

Figura 1. Esquema conciso do caminho delineado pelo idoso até prescrição farmacoterapêutica na rede de saúde. (Fonte: produzida pelos autores)

Os profissionais de saúde abrangidos nesta rede de cuidado com a medicação, como farmacêuticos, gerontólogos, enfermeiros, terapeutas ocupacionais, psicólogos, 
cuidadores, são valiosos para a adesão à terapêutica, importando a discussão da questão com a própria pessoa idosa para empreender esforços que minimizem ou eliminem os aspectos que a impeçam de aderir ao tratamento medicamentoso (SOUSA et al., 2011).

\section{Aporte teórico para a construção do cuidado}

A propósito de vislumbrar a trama de atores envolvida na rede que se configura no processo de adesão ao tratamento farmacológico em idosos é que se retomam algumas perspectivas da ANT, que tem seu primórdio a partir de uma nova forma de pensar a atividade científica, onde o conhecimento gerado passa a ser visto como uma prática social, comportamentos, ações e não apenas resultado de um processo cognitivo excepcional.

Em Lorenzi (2010) tem-se que essa forma de construção do saber científico é chamada construtivismo, onde:

(...) as coisas (sociais, cognitivas, naturais) não são fatos objetivos que a ciência ou entendimento humano percebe e formula, mas sim construções. As coisas, os fatos, os estados psicológicos ou pensamentos sociais seriam, portanto, construções coletivas (p. 47).

Nobre e Pedro (2010) remetem que a ANT se configura com uma visão acerca das práticas cotidianas abarcadas em ciência, tecnologia e sociedade. As conexões de humanos e não humanos configuram redes emaranhadas que permite sua percepção a partir de um processo dinâmico de associações.

Como objetivo a ANT se insere na descrição de uma associação de humanos e não humanos como atores igualmente constituídos, unidos por redes construídas e mantidas a fim de um propósito, incorporando o princípio da simetria generalizada que os torna referidos como actantes (MARCELO, 2012).

Em Gonzales e Baum (2013) observam que na concepção desta teoria o social não deve ser explicado como uma reunião de convicções interligadas, mas acompanhar o movimento dos atores em suas associações, sem determinar a ação e no embate das controvérsias. $O$ interesse está na formação desses movimentos associativos, na busca descritiva do que faz fazer. Para Latour (2012, p. 45):

(...) a ANT sustenta ser possível rastrear relações mais sólidas e descobrir padrões mais reveladores quando se encontra um meio de registrar os vínculos entre quadros de referência instáveis e mutáveis, em vez de tentar estabilizar um deles. 
A atuação na rede é perceptível e os atores não são a fonte da ação, mas foco de várias essencialidades lutando para determinar o agir. Com isso, assumem a ação na rede, não conhecendo quem faz o quê ou que agente aciona o outro, conformando a existência de uma rede heterogênea no choque de controvérsias e ações das entidades, as quais se referem a humanos e não humanos no mundo social (GONZALES; BAUM, 2013).

Segata (2012) destaca que a ideia de associação e de rede não deve ser entendida como sujeitos e objetos relacionados a humanos e não humanos. Ambos agem em uma sucessão de ações distribuídas, não vinculadas a causa e efeito, o que determina a rede como um resultado (e não um dado), a fim de esgotar a descrição dos actantes no evento. Prates (2013) afirma que a construção da imagem de um social em rede ocorre através da especificidade de cada associação, levadas em conta a presença dos atores, as interações e a percepção de que objetos e humanos também são mediadores, ou seja, atuam, transformam, geram significados. A voz dos não humanos deve ser ouvida e isso argumenta a ANT. "Isso, é claro, não implica em pensá-los como intencionais, mas como dotados de alguma subjetividade" (SEGATA, 2012, p. 240). O impacto de um agente na ação de outro se corrobora nas associações de uma rede pela seguinte acepção de Latour (2012, p. 108):

Se você puder, com a maior tranquilidade, sustentar que pregar um prego com ou sem um martelo, ferver água com ou sem uma panela, transportar comida com ou sem um cesto, andar na rua com ou sem roupas, zapear a televisão com ou sem o controle remoto, parar um carro com ou sem o freio, fazer um inventário com ou sem uma lista, administrar uma empresa com ou sem a contabilidade são exatamente as mesmas atividades, que a introdução desses implementos comuns não muda nada "de importante" na realização das tarefas, então você está pronto para visitar a Terra Longínqua do Social e desaparecer daqui. Para todos os outros membros da sociedade esses implementos fazem muita diferença e são, pois, segundo nossa definição, atores - ou, mais exatamente, partícipes no curso da ação que aguarda figuração.

Para Gonzales e Baum (2013) a ANT implica em um uso do social na percepção associativa e que ao ser empregada, metodologicamente, induz a ocorrência de uma mediação entre duas entidades, favorecidas pela ação do ator-rede, o qual:

(...) consiste naquilo que é induzido a agir por uma vasta rede, em forma de estrela, de mediadores que entram e saem. Suas muitas conexões the dão a existência: primeiro os vínculos, depois os atores (LATOUR, 2012, p. 312). 
Cavalcante e Vasconcelos (2007) resgatam estudos que investigam o movimento associativo entre o sistema social e tecnológico para educação em saúde, advindo a ANT como teoria social da tecnologia baseada no aporte de Michel Callon. Evidenciam uma não distinção entre artefatos tecnológicos e estruturas humanas que os utilizam e ao recorrerem à teoria pressupõem a existência de uma rede de atores heterogênea ou sociotécnica, possibilitando ruptura da questão entre valorização excessiva da técnica em detrimento dos processos pedagógicos e políticos, não inferiorizando uma dimensão à outra na rede, além de relações mais simétricas entre sociedade e tecnologia.

Ao trazer os conceitos teóricos abordados para a adesão ao tratamento farmacológico em idosos faz-se necessário definir, no âmbito geral, Redes de Atenção à Saúde, onde Santos et al. (2016, p. 47) aborda como:

(...) um conjunto de ações e serviços articulados, em níveis de complexidade crescentes, com a finalidade de garantir a integralidade da atenção, transversalizando todo o sistema, desenhando o que entendemos por linha de cuidado, que por sua vez, entendemos como o conjunto de saberes, tecnologias e recursos necessários ao enfrentamento de determinados riscos, agravos ou condições específicas do ciclo de vida, a serem ofertados de forma oportuna, articulada e contínua pelo sistema de saúde, entendendo sua implementação como estratégia central da organização e qualificação das redes, com vistas à integralidade do cuidado.

Nesta conceituação percebe-se a ação de vários actantes em relações associativas, em movimentos de confrontos a fim desconstruir uma rede de cuidado em saúde que, transgredida à questão tratada neste trabalho, permite esboçar os agentes envolvidos na trama e suas interações no meio, a fim de delinear os principais pontos na rede que instigam o idoso à ação de aderir ao tratamento com medicamentos.

Os artefatos (bula, receita, comprimido, cápsula, xarope) e os atores que participam desta mediação, são igualmente importantes para que o ato da adesão se efetue, visto que a recepção na unidade de saúde deve estar apta ao acolhimento da pessoa idosa, escutar e encaminhar à consulta; o profissional realizar os exames necessários e ao optar pelo tratamento farmacológico, orientar e esclarecer as dúvidas de forma clara e objetiva; e ao se reportar à farmácia, os agentes financeiros e relacionados à forma farmacêutica a ser 
empregada emergem como ato a ser instruído na dispensação (que traz consigo outra rede de agentes impregnada).

Sousa et al. (2011, p. 177) identifica algumas ações que estimulam o aumento da adesão à terapia, tais como:

(...) prescrever o menor número de medicamentos possível; evitar prescrições sem dias alternados; evitar o fracionamento de comprimidos; explicar para que servem os medicamentos prescritos; pensar nos efeitos secundários sobre outra doença pré-existente; adequar as formas farmacêuticas às capacidades de deglutição do doente; utilizar rótulos legíveis e compreensíveis; aconselhar doentes analfabetos ou com problemas de esquecimento, de visão e de audição, a solicitarem a colaboração de algum familiar ou cuidador; e ainda, esclarecer sobre esquemas posológicos e outras dúvidas, importantes na administração da medicação.

Outros artifícios são apontados, em Brasil (2012), que auxiliam a adesão ao tratamento em idosos, entre os quais: falar em tom de voz um pouco mais elevada e de frente para o indivíduo, pois alguns pacientes podem apresentar perda auditiva; anotar em com letras maiores, usar cores e texturas que identificam os medicamentos evitando risco de troca para usuários com déficit de visão e dispor de caixas organizadoras e associar a tomada do medicamento com a rotina, como horário de refeição ou programa televisivo, para pessoas com dificuldades de memorização.

\section{CONSIDERAÇÓES FINAIS}

$\mathrm{Na}$ busca de articular a rede implicada no encadeamento à aderência farmacoterapêutica na chamada terceira idade argumentou-se em favor da descrição dos vários actantes imbricados, ao empoderar-se da ANT como suporte teórico.

À medida que o pensar ator-rede como perspectiva metodológica desloca a construção do conhecimento de um lugar estático para outro onde tudo (social, humanos e não-humanos) tem voz, alguns elementos como cuidador, médico, farmacêutico, consultório, farmácia, dinheiro, receita, apresentação do medicamento, rotina de vida (horários, televisão, alimentos) são cruciais para influir na adesão ao tratamento pelo idoso pelas muitas atuações possíveis em contexto de contínuas mudanças. 
As vertentes levantadas apontam para a construção de uma trama entrelaçada por várias outras redes que beneficiam o êxito no assentimento à terapia medicamentosa por esse grupo etário, ao congregar rede de atenção em saúde, rede de cuidado psicossocial, rede de suporte familiar, rede de assistência farmacêutica, as quais precisam ser observadas, compreendida o curso de suas movimentações e garantidas como políticas públicas efetivas na esfera da promoção integral à pessoa idosa.

\section{REFERÉNCIAS}

AIOLFI, C. R. et al. Adesão ao uso de medicamentos entre idosos hipertensos. Rev. bras. geriatr. gerontol., Rio de Janeiro, v.18, n.2, p. 397-404, 2015. Disponível em: $<$ http://www.scielo.br/scielo.php?script=sci_arttext\&pid=S1809-

98232015000200397\&lng=en\&nrm=iso>. Acesso em: 5 Jul. 2016.

BRASIL. Conselho Regional de Farmácia do Estado de São Paulo. Dispensação de Medicamentos. Fascículo VIII, Projeto Farmácia Estabelecimento de Saúde. São Paulo, 2012, $100 \mathrm{p}$.

Instituto Brasileiro de Geografia e Estatística, 2013. Disponível em <www.ibge.org.br. $>$ Acesso em junho/2016.

CAVALCANTE, M. T. L.; VASCONCELLOS, M. M. Tecnologia de informação para a educação na saúde: duas revisões e uma proposta. Ciênc. saúde coletiva, Rio de Janeiro, v. 12, n. 3, p. 611-622, 2007.

CINTRA, F. A.; GUARIENTO, M. E.; MIYASAKI, L. A. Adesão medicamentosa em idosos em seguimento ambulatorial. Ciênc. saúde coletiva, Rio de Janeiro, v. 15, supl. 3, p. 3507-3515, Nov. 2010.

GONZALES, Z. K.; BAUM, C. Desdobrando a Teoria Ator-Rede: Reagregando o Social no trabalho de Bruno Latour. Polis e Psique, v. 3, n. 1, 2013. Disponível em: <http://seer.ufrgs.br/index.php/PolisePsique/article/viewFile/36550/26493>. Acesso em: 2 Julho 2016.

LATOUR, B. Reagregando o social: uma introdução à teoria do ator-rede. Salvador: EDUFBA-Edusc, 400 p., 2012.

LORENZI, B. R. Os estudos sociais da ciência: uma relação com o campo CTS. In: Apontamentos de estudos sobre Ciência, Tecnologia \& Sociedade. São Carlos: Pedro \& João Editores, 2010, p. $39-52$.

MARCELO, J. F. Sociologia da ciência: análise bibliométrica da base de dados Scopus. Dissertação (Mestrado em Ciência, Tecnologia e Sociedade). Universidade Federal de São Carlos, Centro de Educação e Ciências Humanas. São Carlos: UFSCar, 2012, 91p. 
NOBRE, J. C. de A.; PEDRO, R. M. L. R. Reflexões sobre possibilidades metodológicas da Teoria Ator-Rede. Cadernos UNIFOA, v. 5, n. 14, 2010.

PRATES, V. Entre formigas e estrelas. Galáxia. (São Paulo, Online), n. 25, p. 206-210, jun. 2013. Disponível em: <http://www.scielo.br/pdf/gal/v13n25/v13n25a17.pdf>. Acesso em: 2 Jul. 2016.

SANTOS, C. T. B. dos et al. Percurso do idoso em redes de atenção à saúde: um elo a ser construído. Physis, Rio de Janeiro, v. 26, n. 1, p. 45-62, 2016. Disponível em:<http://www.scielo.br/scielo.php?script=sci_arttext\&pid=S0103-

73312016000100045\&lng=en\&nrm=iso>. Acesso em: 5 Jul. 2016.

SEGATA, J. Resenha. LATOUR, B. Reagregando o Social: uma introdução à Teoria do Ator-Rede. Universidade Federal de Santa Catarina. Ilha Revista de Antropologia. v. 14, n. 1,2, 2012.

SOUSA, S.et al. Polimedicação em doentes idosos: adesão à terapêutica. Ver Port Clin Geral, Lisboa, v. 27, n. 2, p. 176-182, 2011. Disponível em $<$ http://www.scielo.mec.pt/scielo.php?script=sci_arttext\&pid=S087071032011000200005\&lng=pt\&nrm=iso >. Acesso em: 5 jul. 2016.

TAVARES, N. U. L. et al. Fatores associados à baixa adesão ao tratamento medicamentoso em idosos. Rev. Saúde Pública, São Paulo, v. 47, n. 6, p. 1092-1101, 2013. 